\title{
One-channel Ka-band pulse compressor
}

\author{
S. V. Kuzikov, ${ }^{1,2}$ A. A. Vikharev, ${ }^{1,2}$ M. E. Plotkin, ${ }^{1,2}$ D. Yu. Shegol'kov, ${ }^{2}$ and J. L. Hirshfield ${ }^{1,3, *}$ \\ ${ }^{1}$ Omega-P, Inc., 199 Whitney Avenue, New Haven, Connecticut 06511, USA \\ ${ }^{2}$ Institute of Applied Physics RAS, 46 Ulyanov Street, 603600, Nizhny Novgorod, Russia \\ ${ }^{3}$ Yale University, 272 Whitney Avenue, New Haven, Connecticut 06520, USA
}

(Received 19 June 2007; published 9 August 2007)

\begin{abstract}
A compact rf pulse compressor, fed by a phase-modulated pulse, is described for producing high-power flattop pulses at Ka-band. The compressor consists of a single axisymmetrical cavity operating in a combination of $\mathrm{TE}_{0 n}$ modes $(n=1,2,3)$, that have zero electric fields at the walls. This feature enhances the breakdown strength of the system and makes it possible to increase the output power, as compared with a traditional SLED-II pulse compressor. Advantages include use of one channel instead of two, and no requirement for a 3-dB hybrid coupler. This $34 \mathrm{GHz}$ one-channel pulse compressor (OC SLED-II) is designed to multiply peak power produced by the Yale/Omega-P Ka-band magnicon amplifier (30$40 \mathrm{MW}, 0.5-1 \mu \mathrm{s}$ ) by (3-4):1 and to compress in time by (5-6):1. The efficiency of the proposed compressor is similar to that of SLED-II. Results of low-power tests carried out on a $30 \mathrm{GHz}$ prototype of the compressor are discussed.
\end{abstract}

DOI: 10.1103/PhysRevSTAB.10.082001

PACS numbers: $84.40 . \mathrm{Az}$

\section{INTRODUCTION}

Development of high-gradient accelerating structures for a possible future normal-conducting $e^{-}-e^{+}$linear collider requires rf sources at frequencies in the range of $10-34 \mathrm{GHz}$ [1]. These sources are necessary in order to conduct basic research and development $(R \& D)$ on mechanisms that limit rf electric and magnetic field strengths at material surfaces, and to conduct full-scale testing of the accelerating structures. The ultimate goal of this endeavor is to develop new accelerator structures that can reliably sustain working acceleration gradients above $\sim 100 \mathrm{MeV} / \mathrm{m}$. Typical required powers for such tests fall in the range of 100-200 MW with pulse widths of $100-200 \mathrm{~ns}$. This power level can be achieved by creation of a longer pulse at an rf source and then employing an $\mathrm{rf}$ pulse compressor that transforms the long pulse into a shorter pulse of higher power.

A multimode SLED-II pulse compressor illustrated schematically in Fig. 1 has recently been developed, originally as a prospective system at X-band for NLC and JLC $[2,3]$. This device remains relevant as a core element in a test facility to produce the high peak powers required for high-gradient R\&D. This SLED-II compressor consists of two identical resonant delay lines which are fed by an input rf pulse by means of the coupling holes. The delay lines are connected through a 3-dB hybrid coupler which allows backward reflection to the input to be avoided under the condition that the cavities are indeed identical. Compression occurs due to frequency dispersion of the cavities

\footnotetext{
*Corresponding author
} jay.hirshfield@yale.edu and phase modulation of the input $\mathrm{rf}$ pulse. In tests using four $50 \mathrm{MW}$ klystrons, $400 \mathrm{~ns}$ wide rf pulses of greater than $500 \mathrm{MW}$ peak power were produced [3].

One of the indisputable advantages of the SLED-II compressor is the flattop output pulse that it can produce, which is desirable for traveling-wave structure tests. The flattop pulse is formed through use of long delay lines which use oversized waveguides. The delay lines operate in the low-loss breakdown-proof $\mathrm{TE}_{01}$ mode or $\mathrm{TE}_{01}-\mathrm{TE}_{02}$ mode combinations [3].

One drawback of the classical SLED-II pulse compressor is its requirement for two long, identical, delay lines. Previous attempts to improve design of the classical SLED-II through the use of two-, three-, or even fourmode structures were directed on a reduction of lengths of the delay lines $[3,4]$. Still, a $3-\mathrm{dB}$ hybrid coupler is also required in these schemes, and may be considered a serious challenge for a millimeter-wave SLED-II pulse compressor, because of the impossibility at the high-power levels of using single mode rectangular waveguide. Thus, there is the need for a compact, reflection-free, "breakdownproof" design of a pulse compressor providing flattop output pulses. Such a design is described in this paper.

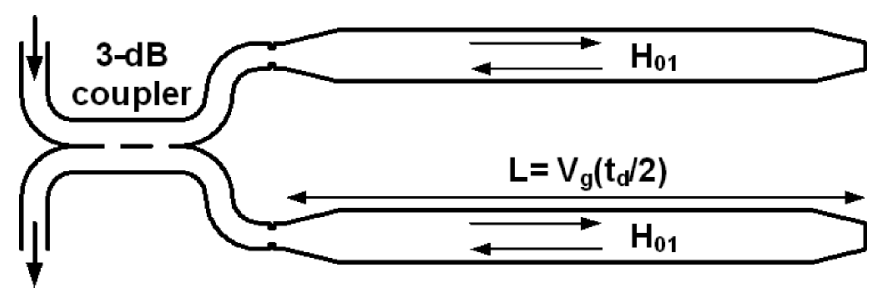

FIG. 1. Classical SLED-II pulse compressor. 


\section{DETAILED ANALYSIS OF ONE-CHANNEL COMPRESSION SCHEME}

A schematic drawing of a novel pulse compressor is shown in Fig. 2. It is an axisymmetric multimode cavity which operates as a reflection-free multipass travelingwave structure [5,6]. This is illustrated in Fig. 3. The traveling-wave eigenmode in the pulse compressor consists of the $\mathrm{TE}_{02}$ mode propagating one way (left-to-right in Fig. 2) and the $\mathrm{TE}_{03}$ mode propagating the other way (right-to-left in Fig. 2). Mutual mode converters (elements 1 in Fig. 2) at each end of the cavity convert one of these modes into the other by means of selective reflecting converters. The input (output) wave is in the $\mathrm{TE}_{01}$ mode, and enters (leaves) at the left (right). The left-toright traveling $\mathrm{TE}_{01}$ mode is coupled only with the left-toright traveling $\mathrm{TE}_{02}$ mode by means of a selective mode converter (element 2) placed in the middle of the cavity. This converter plays the role of an input coupler and must provide the optimal level of conversion $\mathrm{TE}_{01} \rightarrow \mathrm{TE}_{02}$ in order to obtain high compression efficiency. The right-toleft traveling $\mathrm{TE}_{03}$ mode must not be perturbed by the $\mathrm{TE}_{01} \rightarrow \mathrm{TE}_{02}$ coupling converter.

The feeding $\mathrm{TE}_{01}$ wave propagates to the $\mathrm{TE}_{01} \rightarrow \mathrm{TE}_{02}$ mode coupler, where a part of the incident power is converted into the forward $\mathrm{TE}_{02}$ wave. The $\mathrm{TE}_{02}$ wave then propagates to the right $\mathrm{TE}_{02} \leftrightarrow \mathrm{TE}_{03}$ reflecting converter and is completely transformed into the $\mathrm{TE}_{03}$ wave, which propagates to the left end reflector without change. At the left end the $\mathrm{TE}_{03}$ mode has full conversion into the forward $\mathrm{TE}_{02}$ mode. At the $\mathrm{TE}_{01} \rightarrow \mathrm{TE}_{02}$ mode converter this wave is added constructively to a new portion of power incident in the feeding forward $\mathrm{TE}_{01}$ mode. So, like in any SLED-II

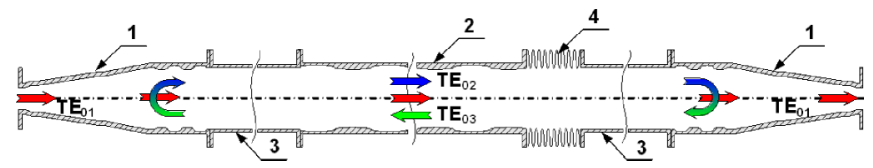

FIG. 2. (Color) One-channel SLED-II pulse compressor. 1, $\mathrm{TE}_{02} \leftrightarrow \mathrm{TE}_{03}$ reflective mode converter; $2, \mathrm{TE}_{01} \rightarrow \mathrm{TE}_{02}$ transmission mode converter; 3 , waveguide delay lines; 4 , bellows.

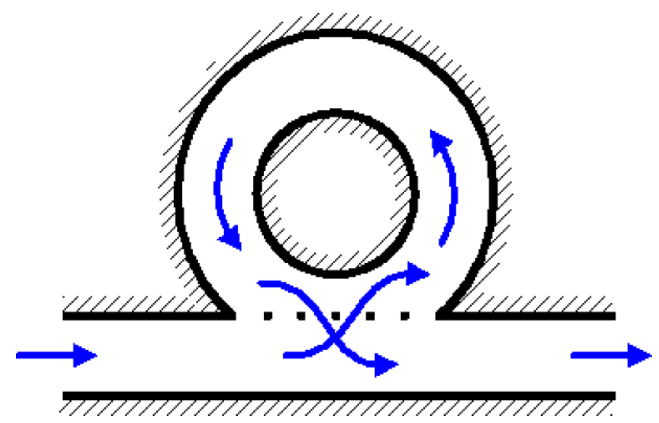

FIG. 3. (Color) An equivalent scheme based on traveling-wave cavity and directional coupler. pulse compressor, rf power builds up inside the compressor. The transmitted signal in a form of the $\mathrm{TE}_{01}$ mode decreases, so long as the input phase remains constant. When a $180^{\circ}$ phase flip is imposed on the incoming wave, the compressor's stored energy begins to leak out, being in the same phase as that of the incoming $\mathrm{TE}_{01}$ power. This amounts to an emissive burst of rf power at the output. This amounts to pulse compression. From this point of view, the suggested compressor works like a SLED-II compressor and all the theory created for SLED-II is therefore applicable $[2,3]$.

Obviously, the desired output pulse duration of the compressor $\tau_{\text {out }}$ depends on the length $l$ of the cavity, i.e.

$$
\tau_{\mathrm{out}}=\tau_{2}+\tau_{3} \approx l\left(v_{2}+v_{3}\right) / v_{2} v_{3}
$$

where $\tau_{2}$ and $\tau_{3}$ are one-way group delays, and $v_{2}$ and $v_{3}$ are group velocities of the $\mathrm{TE}_{02}$ and $\mathrm{TE}_{03}$ modes, respectively. This duration is connected with the duration of the input pulse $\tau_{\text {in }}$ by means of compression ratio coefficient $C_{r}$, namely $\tau_{\text {out }}=\tau_{\text {in }} / C_{r}$. The power gain $G=P_{\text {out }} / P_{\text {in }}$ is defined as the ratio of output power to incident power. The efficiency is the ratio of energy concentrated in time interval of length $\tau_{\text {out }}$ to the total rf energy of incident pulse.

It is assumed that both the $\mathrm{TE}_{02} \leftrightarrow \mathrm{TE}_{03}$ reflective mutual mode converter and the $\mathrm{TE}_{01} \rightarrow \mathrm{TE}_{02}$ selective mode coupler must be broadband enough to accommodate the pulse spectrum width $\Delta \omega$, which is approximately equal to $\Delta \omega=2 \pi / \tau_{\text {out }}$.

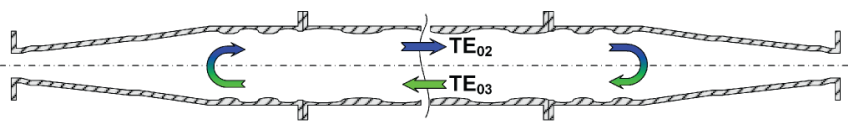

(a)

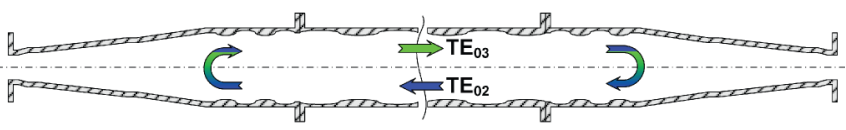

(b)

FIG. 4. (Color) $\mathrm{TE}_{02}$ (forward)- $\mathrm{TE}_{03}$ (backward), the operating eigenmode. Spurious $\mathrm{TE}_{03}$ (forward)- $\mathrm{TE}_{02}$ (backward), the degenerate eigenmode.

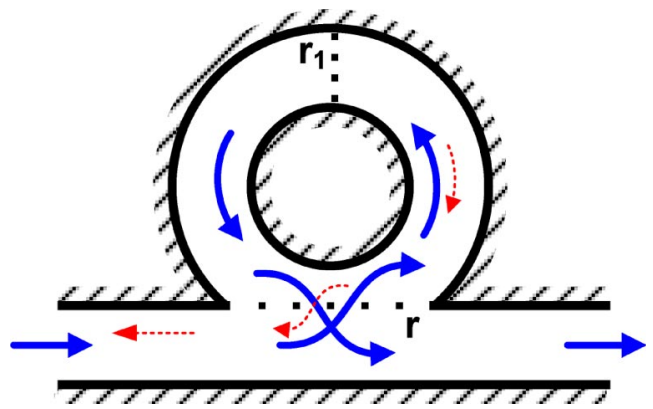

FIG. 5. (Color) An equivalent scheme to explain coupling of eigenmodes of opposite rotations. 


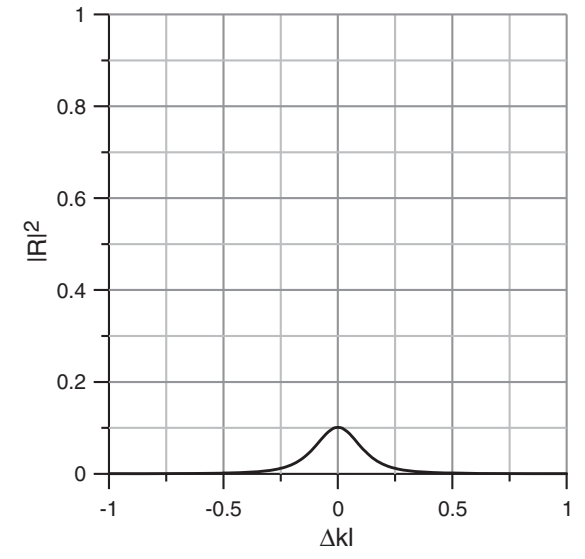

(a)

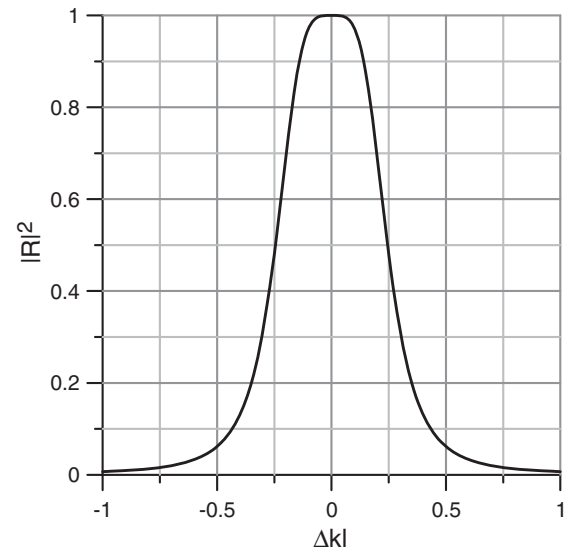

(b)

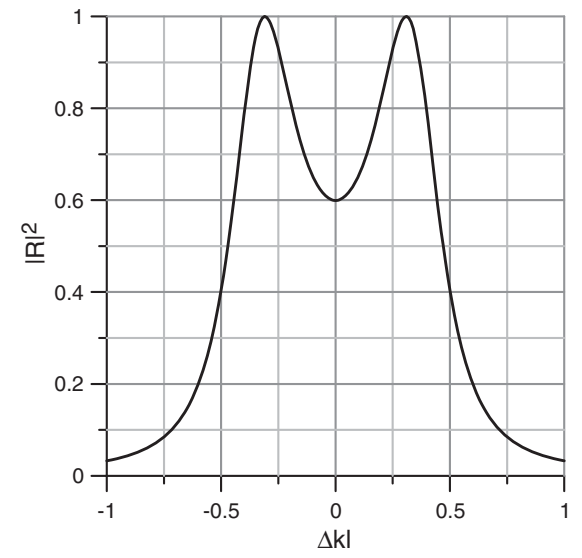

(c)

FIG. 6. Spurious reflection caused by mutual coupling of the operating and spurious degenerate eigenmodes: (a) coupling is small; (b) coupling is critical; (c) coupling is too big.

In order to allow fine frequency tuning of the compressor, to compensate for thermal fluctuations, for example, there is a bellows (element 4 in Fig. 2) which allows mechanical changes in the length of the cavity. The bellows's corrugation period is to be small in comparison with a half wavelength and will therefore not perturb mode propagation in the cavity.

Among eigenmodes of the cavity there, of course, exists a degenerate mode that consists of the same mixture of $\mathrm{TE}_{02}$ and $\mathrm{TE}_{03}$ modes, but with directions of propagation opposite to those of the operating eigenmode. The two degenerate modes are depicted in Figs. 4(a) and 4(b) correspondingly. The spurious eigenmode has the potential to be excited from imperfect design of the mode converters, for example, if one wave is converted into another desired mode with less than full $100 \%$ efficiency. This leads to reflection of some rf power and splitting of eigenfrequencies.

This reasoning suggests a simple equivalent scheme, depicted in Fig. 5, which helps to describe the mentioned phenomena. As shown in Fig. 5, coupling of the degenerate eigenmodes of the opposite direction of travel is modeled by an additional reflector placed in the middle. This reflector with reflection coefficient $r_{1}$ causes excitation of the spurious eigenmode which leaks through the directional coupler in backward direction forming reflection. Based on the model shown in Fig. 5, one can easily write a formula for the reflection coefficient using coupling factor $r$ of the directional coupler and spurious coupling factor $r_{1}$ of the inserted reflector, namely

$|R|^{2}=\frac{r^{4} \cdot r_{1}^{2}}{\left|e^{-i 2 k l}-2 \sqrt{\left(1-r^{2}\right) \cdot\left(1-r_{1}^{2}\right)}+\left(1-r^{2}\right) \cdot e^{i 2 k l}\right|^{2}}$.

An analysis of Eq. (2) shows the existence of a critical value of the spurious coupling factor between eigenmodes if we assume that $r$ is fixed, as shown in Fig. 6. In particular, when spurious coupling is small enough, reflection is negligible [Fig. 6(a)]. If one increases spurious coupling, the reflected power reaches $100 \%$ [Fig. 6(b)] at a resonant frequency given by condition $2 k l=2 \pi n$ ( $n$ is a positive integer). If one continues to increase coupling between degenerated eigenmodes, Wien's splitting [7] of frequencies occurs [Fig. 6(c)].

The critical value of coupling can be found from the condition for reflection's extremum (at resonant condition $\Delta k \cdot l=0)$ as a function of $r_{1}$ :

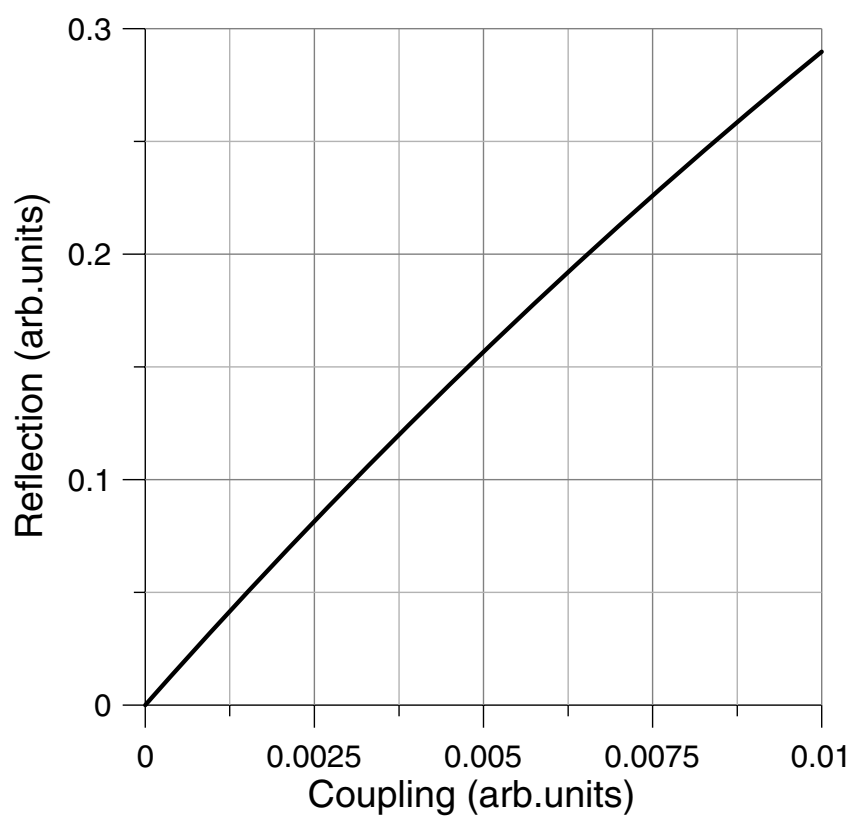

FIG. 7. Reflection of compressor vs spurious coupling factor of degenerate eigenmodes. 


$$
\frac{\partial|R|^{2}}{\partial r_{1}}=0
$$

which leads to the simple formula for the mentioned critical coupling factor:

$$
r_{1 \mathrm{cr}}^{2}=\frac{r^{2}}{2-r^{2}}
$$

For example, if $r^{2}=0.5$ (optimal value for compression ratio $C_{r}=6$ ), then $r_{1 \mathrm{cr}}^{2}=33 \%$. In order to provide reflection at level $\sim 1 \%$ in this case, spurious coupling between the operating eigenmode and the degenerate spurious mode should be not more than $0.1 \%$, as follows from Fig. 7, where reflection is plotted as a function of spurious coupling factor. That is why all further calculations of all compressor's components were aimed to provide diffraction losses at level less than $0.1 \%$.

\section{TE 02 $\leftrightarrow$ TE $_{03}$ REFLECTIVE MODE CONVERTER}

This mode converter consists of several sections, as shown in Fig. 8. The $\mathrm{TE}_{02}$ mode is incident from the left. In the first corrugated section, the incident mode is converted into a mixture of $50 \% \mathrm{TE}_{03}$ and $50 \% \mathrm{TE}_{02}$. To achieve this, a corrugation period given by

$$
D_{1}=2 \pi /\left(h_{2}-h_{3}\right)
$$

is chosen, where $h_{2}$ and $h_{3}$ are propagation constants of the $\mathrm{TE}_{02}$ and $\mathrm{TE}_{03}$ modes, respectively. The next part is a narrowed section which totally reflects the $\mathrm{TE}_{03}$ mode because the following cylindrical section is cut off for the $\mathrm{TE}_{03}$ mode. The $\mathrm{TE}_{02}$ mode propagates through this section while accumulating a phase shift. This $\mathrm{TE}_{02}$ mode is reflected by the next down taper. Therefore, in the corrugated section the returning $50 \% \mathrm{TE}_{02}$ mode is converted into $\mathrm{TE}_{03}$ mode, and merges completely with the first $50 \%$ $\mathrm{TE}_{03}$ mode due to proper phase differences provided by choice of a proper length for the aforementioned cylindrical section. This idea of a $\mathrm{TE}_{02}-\mathrm{TE}_{03}$ mode converter is very similar to that suggested in [4].

The $30 \mathrm{GHz}$ mode converter was calculated to have an input diameter of $40 \mathrm{~mm}$ and an output diameter $15 \mathrm{~mm}$. A conversion efficiency of $99.9 \%$ is achievable, as shown

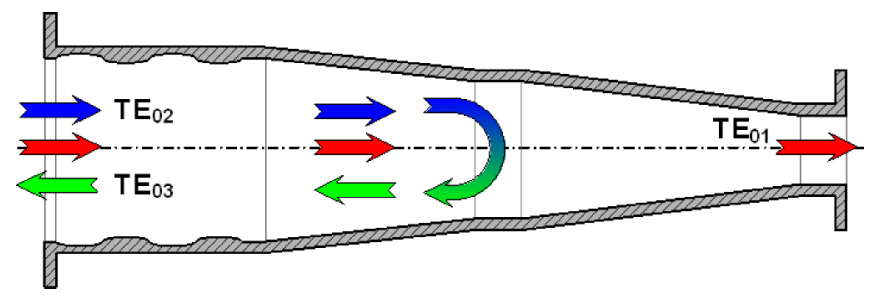

FIG. 8. (Color) $\mathrm{TE}_{02} \leftrightarrow \mathrm{TE}_{03}$ mode converter-reflector.

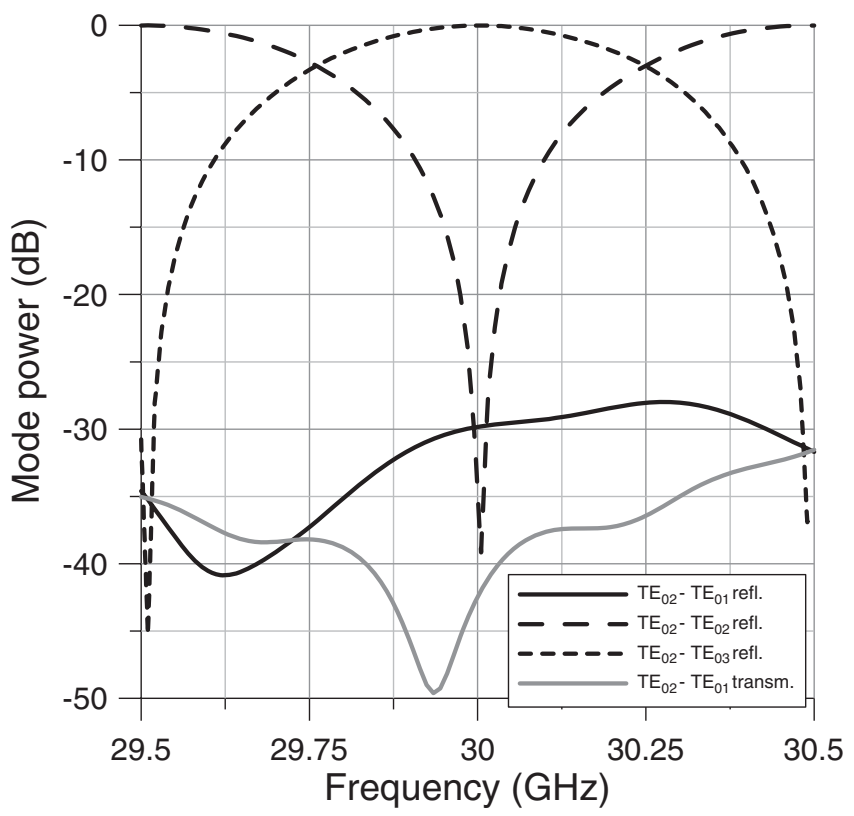

(a)

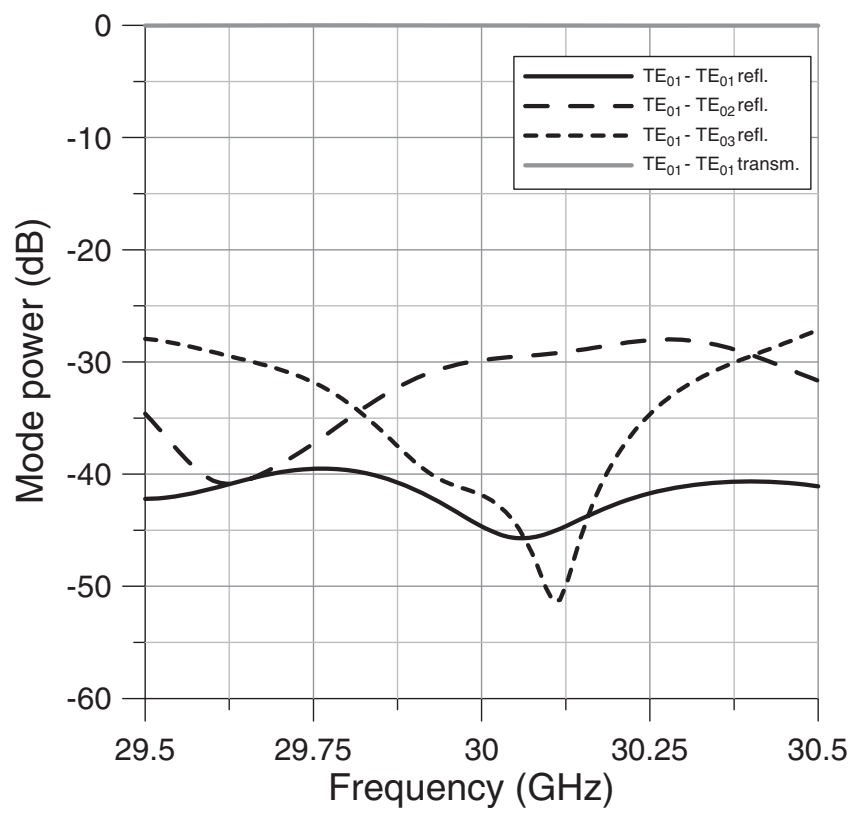

(b)

FIG. 9. Results of calculation of mode conversions in the $\mathrm{TE}_{01} \leftrightarrow \mathrm{TE}_{02}$ mode reflecting converter: (a) with $\mathrm{TE}_{02}$ mode incidence; (b) with $\mathrm{TE}_{01}$ mode incidence.

in Fig. 9. The $\mathrm{TE}_{01}$ mode is also transmitted by the converter with high efficiency (diffraction losses are $\sim 0.1 \%$ ). Note that the frequency bandwidth of this component is $250 \mathrm{MHz}$ at $90 \%$ power level. This means that the required time to obtain steady state operation of this component is not less than $5 \mathrm{~ns}$. In fact, the mentioned time prohibits obtaining of output pulses which are shorter than $5 \mathrm{~ns}$, because significant distortion of the pulse shape will occur for shorter pulses. 


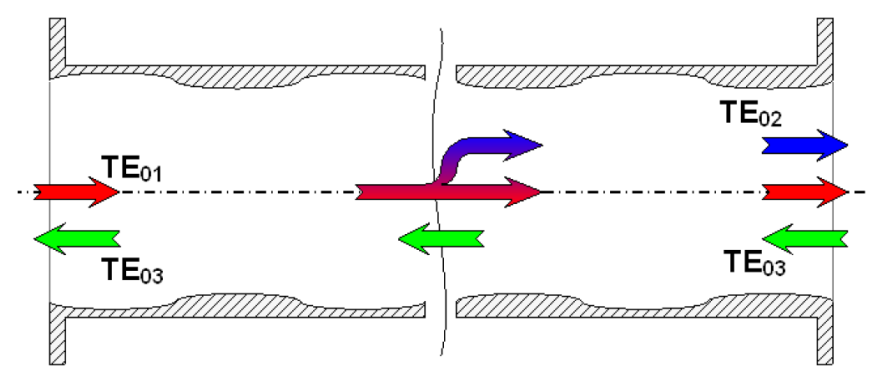

FIG. 10. (Color) $\mathrm{TE}_{01}-\mathrm{TE}_{02}$ selective directional coupler.

\section{TE $_{01} \rightarrow$ TE $_{02}$ MODE CONVERTER}

This element is depicted in Fig. 10. It plays the role of a directional coupler, satisfying three conditions simultaneously, namely (i) the forward $\mathrm{TE}_{01}$ mode should provide necessary coupling with the forward $\mathrm{TE}_{02}$ mode (for example, for $C_{r}=6$ the conversion must be 50\%) without losses into other modes; (ii) the backward $\mathrm{TE}_{03}$ mode should propagate without diffraction losses; and (iii) the forward $\mathrm{TE}_{02}$ mode should not have significant scattering into other modes except the forward $\mathrm{TE}_{01}$ mode. Note that condition (iii) is satisfied automatically when conditions (i) and (ii) are satisfied. In order to satisfy all three conditions, the profile $r(z)$ was taken to have the form:

$$
\begin{aligned}
r(z)= & r_{1} \sin \left[\left(h_{1}-h_{2}\right) z+\varphi_{1}\right]+r_{2} \sin \left[\left(h_{2}-h_{3}\right) z+\varphi_{2}\right] \\
& +r_{3} \sin \left[\left(h_{1}-h_{3}\right) z+\varphi_{3}\right],
\end{aligned}
$$

as a function of coordinate $z$, where $h_{1}, h_{2}$, and $h_{3}$ are propagation constants of the $\mathrm{TE}_{01}, \mathrm{TE}_{02}$, and $\mathrm{TE}_{03}$ modes, respectively. The corrugation period of the main profile space harmonic with amplitude $r_{1}$ and period $D_{2}=$ $2 \pi /\left(h_{1}-h_{2}\right)$ is responsible for transformation of the input $\mathrm{TE}_{01}$ mode into the $\mathrm{TE}_{02}$ mode. Magnitudes of other space harmonics with amplitudes $r_{2}$ and $r_{3}$ are to be optimized in order to suppress scattering into other undesirable modes.

The described $\mathrm{TE}_{01}-\mathrm{TE}_{02}$ coupler with an average diameter of $40 \mathrm{~mm}$ was designed for a coupling factor of $50 \%$ at $30 \mathrm{GHz}$. As seen in Figs. 11(a) and 11(b), mutual conversion of the $\mathrm{TE}_{01}$ and $\mathrm{TE}_{02}$ modes is very close to the desired level of $50 \%$. Spurious scattering is negligibly small. A frequency bandwidth of $1 \mathrm{GHz}$ is 4 times larger than the bandwidth of the $\mathrm{TE}_{02}-\mathrm{TE}_{03}$ reflecting converter.

\section{EXAMPLE OF A 34 GHZ PULSE COMPRESSION SYSTEM}

The most natural scheme to constitute an OC-SLED-II is presented in Fig. 12(a), where the $34 \mathrm{GHz}$ magnicon amplifies a low-power rf signal which has a sudden phase modulation imposed in a form of a $180^{\circ}$ phase flip. This amplified pulse with its phase modulation feeds the OCSLED-II compressor. The considered $50 \% \mathrm{TE}_{01} \rightarrow \mathrm{TE}_{02}$ conversion in the coupler that was chosen for the example

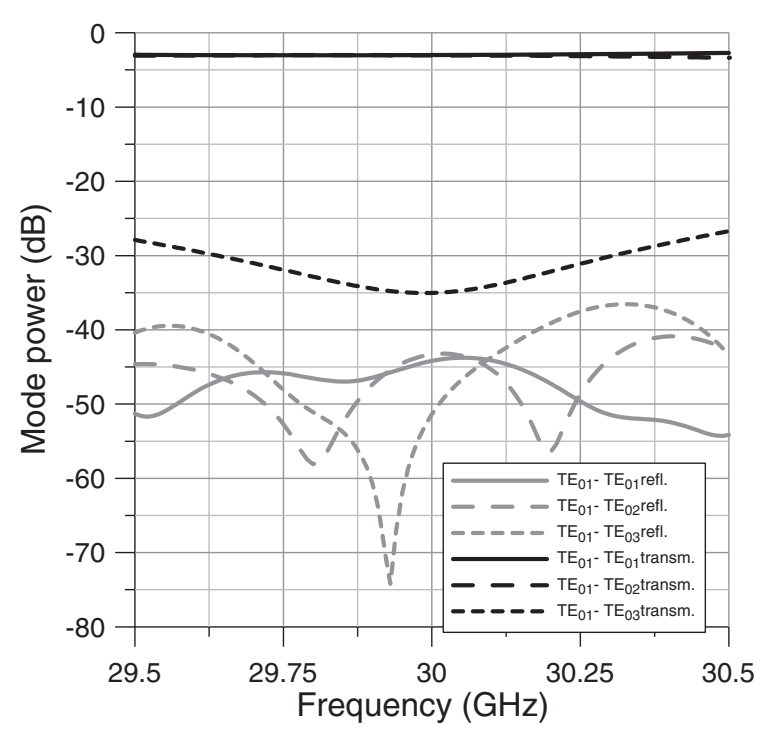

(a)

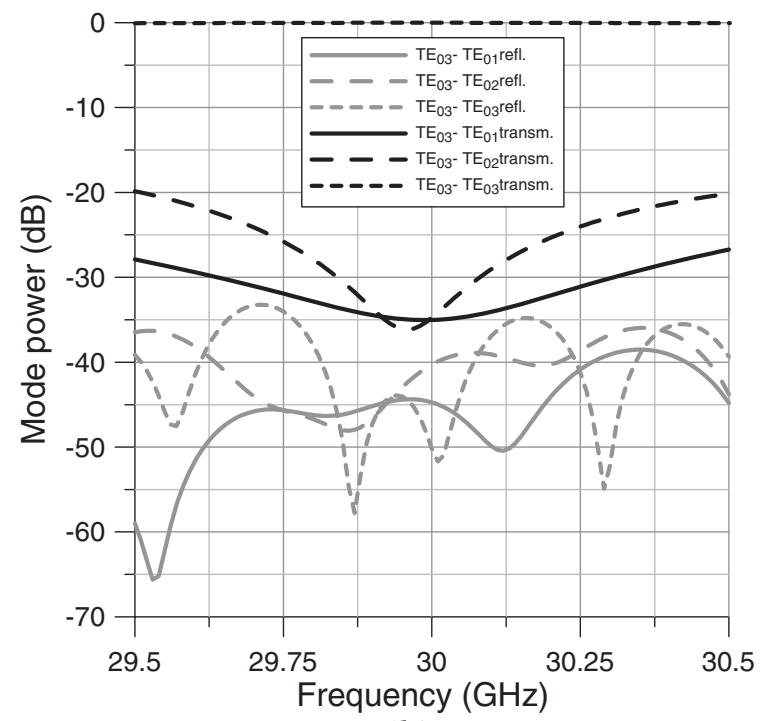

(b)

FIG. 11. Mode conversion at $\mathrm{TE}_{01}-\mathrm{TE}_{02}$ selective directional coupler: (a) left-to-right $\mathrm{TE}_{01}$ mode is incident; (b) right-to-left $\mathrm{TE}_{03}$ mode is incident.

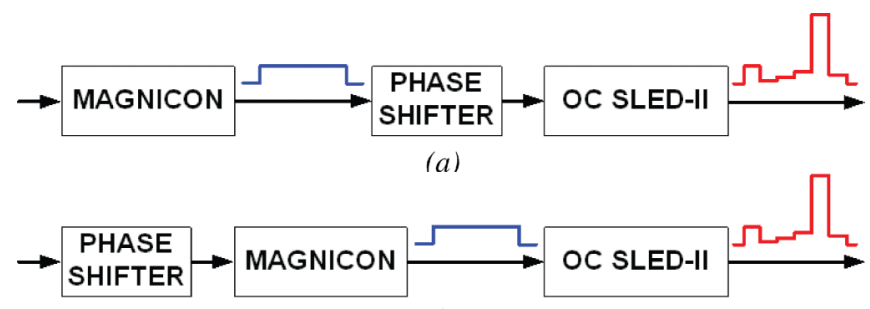

(b)

FIG. 12. (Color) Operation of OC SLED-II with a $180^{\circ}$ phase flip: (a) phase shifter is installed at the input of the magnicon; (b) phase shifter is installed behind the magnicon. 


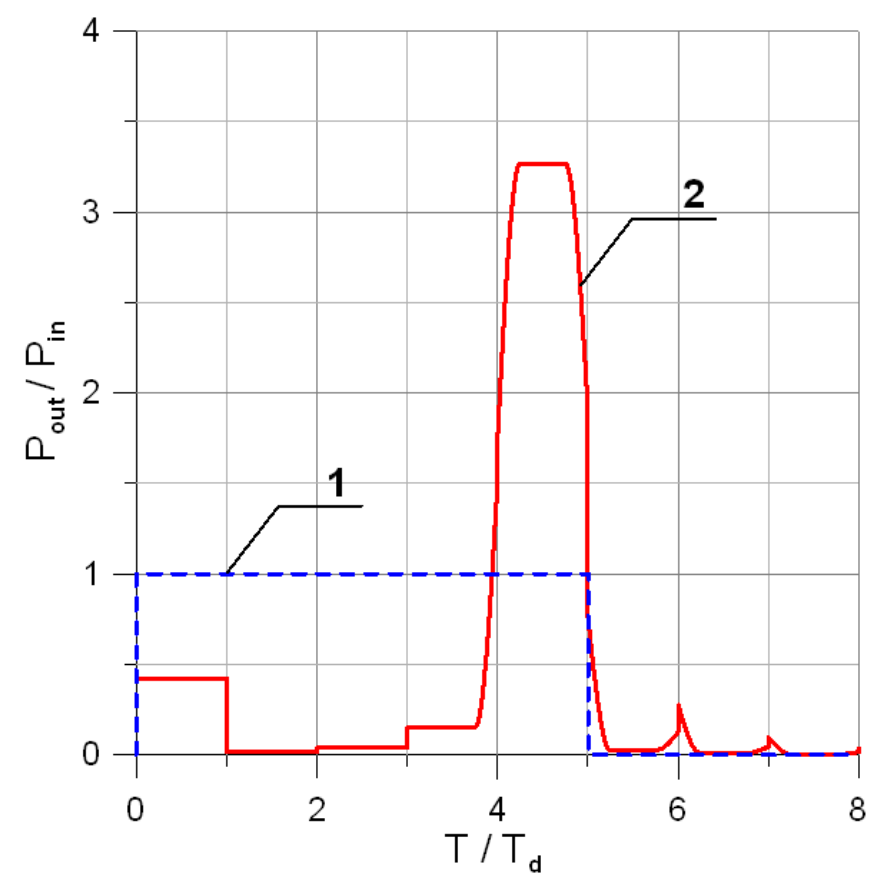

FIG. 13. (Color) Calculated envelope of the input (1) and output (2) pulses for the one-channel SLED-II with phase reversal switch time of $50 \mathrm{~ns}, \tau_{\text {out }}=T_{d}=100 \mathrm{~ns}, C_{r}=5$.

given in Sec. IV gives rise to a power gain of 4 times and $80 \%$ ideal (lossless) efficiency.

The Yale/Omega-P 34-GHz magnicon has come into operation, with performance approaching its design ratings of 35-40 MW peak power output in $1.0 \mu$ s pulses, at a $10 \mathrm{~Hz}$ repetition rate [8]. The magnicon frequency bandwidth is restricted, in particular, by high $Q$-factors of operating magnicon's cavities. As a result, a too fast phase reversal in the input pulse cannot be amplified, and a notch will result in the output pulse. In order to simulate pulse compression with noninstantaneous phase reversal taken into account, we have calculated the compression with phase reversal during a $50 \mathrm{~ns}$ interval that corresponds to the magnicon bandwidth [8]. The resulting output pulse envelope is shown in Fig. 13. In spite of a long transition time which is only 2 times less than the output pulse duration, the resulted pulse shape has a $50 \mathrm{~ns}$ flattop and seems acceptable for accelerating structure tests.

TABLE I. Design parameters of the $34 \mathrm{GHz}$ OC SLED-II pulse compressor.

\begin{tabular}{lc}
\hline \hline Operating mode & $\mathrm{TE}_{01} / \mathrm{TE}_{02} / \mathrm{TE}_{03}$ \\
Compression ratio & 6 \\
Power gain & 3.6 \\
Length of delay lines & $1 \times 11.5 \mathrm{~m}$ \\
One-trip losses & $15 \%$ \\
Reflection & $1 \%$ \\
Input pulse & $25 \mathrm{MW} \times 600 \mathrm{~ns}$ \\
Output pulse & $90 \mathrm{MW} \times 100 \mathrm{~ns}$ \\
Efficiency & $60 \%$ \\
\hline \hline
\end{tabular}

The alternative scheme [Fig. 12(b)] does not require a broad bandwidth rf source, but a phase shifter has to be operated at a high-power level. Such a high-power component, in principle, can be based on photoconductive semiconductor switches controlled by laser light [9], or plasma switches [10], or ferroelectrics to be switched by a highvoltage pulse [11].

As an illustrative example, Table I gives parameters of a one-channel SLED-II pulse compressor suitable for operation at $34 \mathrm{GHz}$, based on principles describe in this paper.

\section{LOW-POWER TESTS}

A $30 \mathrm{GHz}$ prototype of the one-channel compressor was built in accordance with calculation results described above. The tests were aimed to demonstrate key principles of operation [6]. The investigated compressor included two $\mathrm{TE}_{02} \leftrightarrow \mathrm{TE}_{03}$ reflectors, a $\mathrm{TE}_{01} \rightarrow \mathrm{TE}_{02}$ mode converter, and a waveguide section of variable length for fine tuning, as shown in Fig. 14. Each reflector also had a central section in a form of a movable plunger which allowed

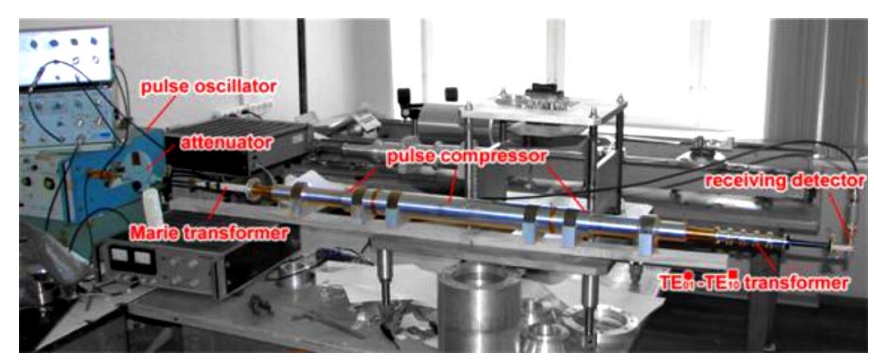

FIG. 14. (Color) Photograph of the $30 \mathrm{GHz}$ prototype.

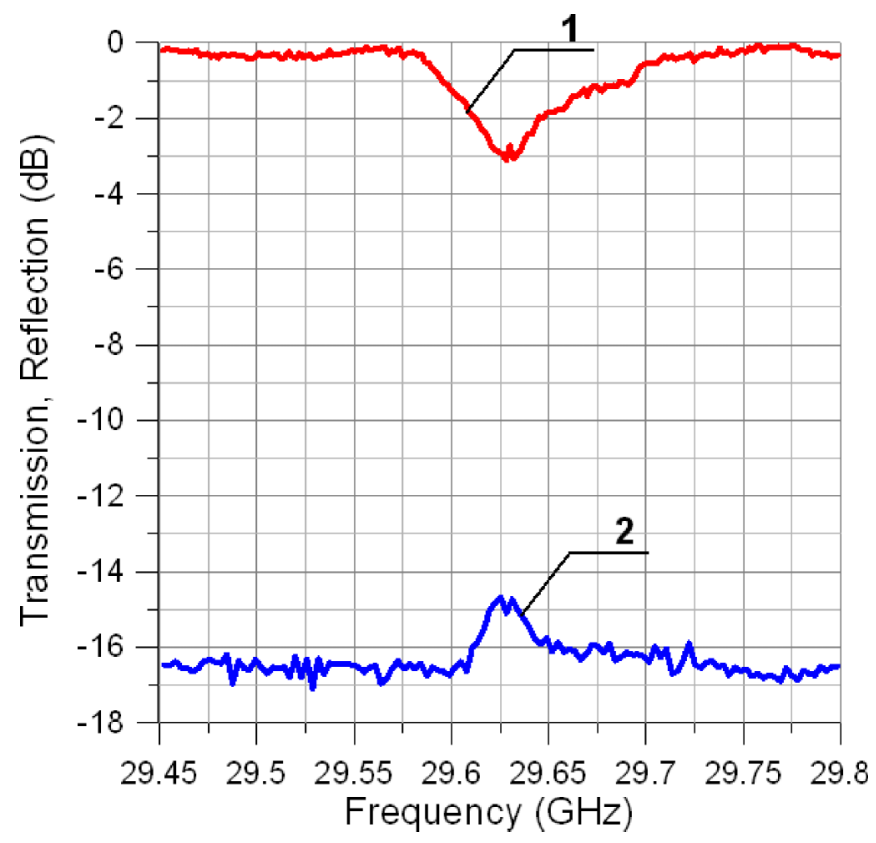

FIG. 15. (Color) Transmission (curve 1) and reflection (curve 2) of the tested pulse compressor. 
TABLE II. Parameters of the tested $30 \mathrm{GHz}$ OC SLED-II prototype.

\begin{tabular}{lcc}
\hline \hline Parameters & Calculation & Measurement \\
\hline Frequency, GHz & 30 & $29.6-30$ \\
$Q_{\text {ohm }}$ & 25000 & $2 \times 10^{4}$ \\
$Q_{\text {load }}$ & 2300 & 2000 \\
$C_{r}$ & 7 & $6-7$ \\
Power gain $G$ & 4.5 & $3-4.5$ \\
Reflection $R, \%$ & 1 & 3 \\
Input pulse duration, ns & 70 & $80-85$ \\
Output pulse duration, ns & 10 & 12.5 \\
Efficiency, $\%$ & 64 & $>60$ \\
\hline \hline
\end{tabular}
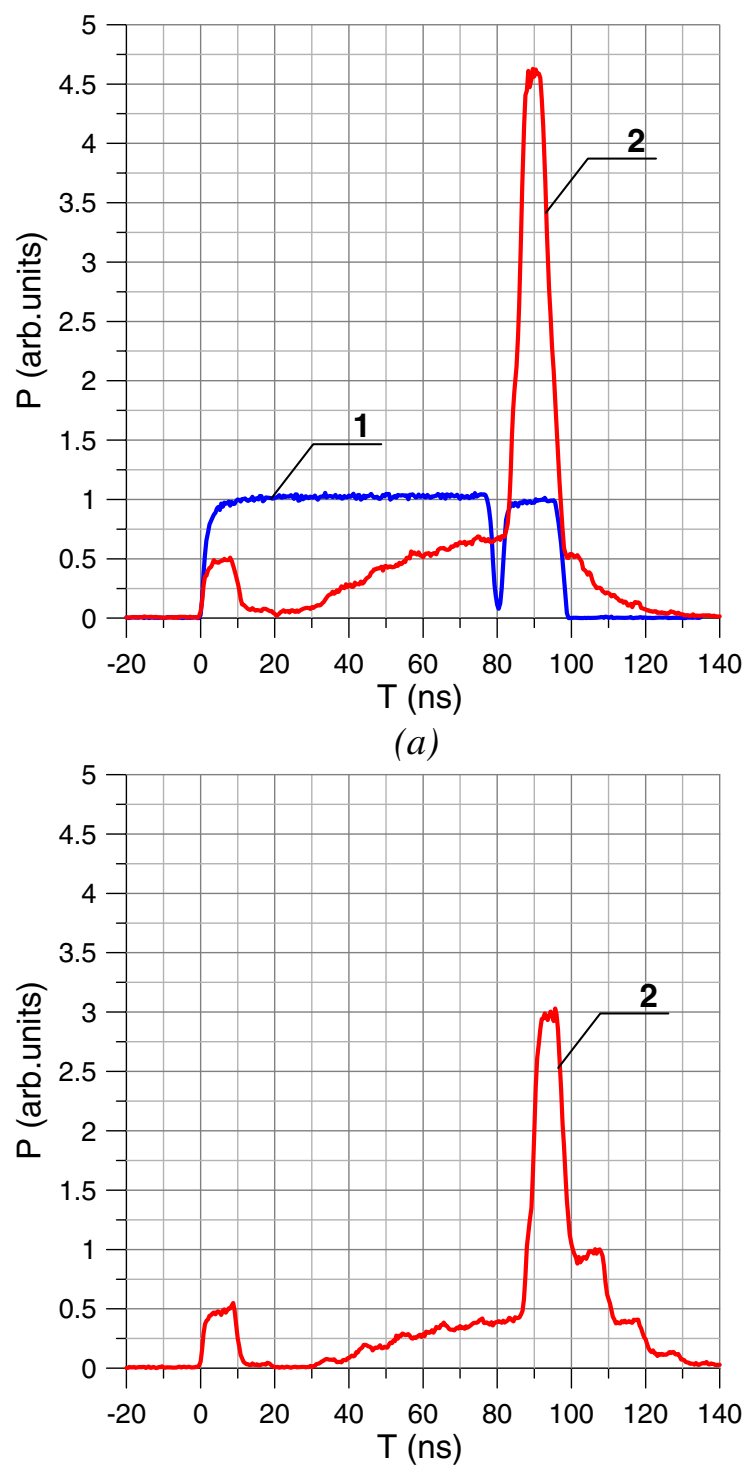

(b)

FIG. 16. (Color) Oscillograms of the incident (curve 1) and compressed (curve 2) pulses in a case of $180^{\circ}$ phase flip: (a) $f=$ $30 \mathrm{GHz}, C_{r}=6.5, G=4.5$; (b) $f=29.6 \mathrm{GHz}, C_{r}=7, G=3$. changes in the mutual phase of the $\mathrm{TE}_{02}-\mathrm{TE}_{03}$ modes and, thus, to tune the conversion efficiency. To simplify the first test, long delay line waveguides were excluded, and the total length of the compressor was $1200 \mathrm{~mm}$. Thus, taking into account group delays, the resulting round-trip delay time was about $13 \mathrm{~ns}$. The rf power was delivered to the compressor by means of a Marie mode transformer, and at the output of the compressor a so-called serpentlike $\mathrm{TE}_{01}-\mathrm{TE}_{11}$ mode converter was used. First of all, we measured reflection from the whole compressor at the operating frequency, tuning flexible parts of reflectors at optimal positions. As a result (as seen in Fig. 15), the reflection maximum did not exceed $3 \%$, a value that is close to reflection from the Marie transformer alone. The loaded $Q$-factor was approximately $Q_{l}=2000$.

From the experimental data, round-trip losses were estimated to be $\sim 5 \%$, which could be due to the brass movable plungers in the end reflectors. Other main parameters of the tested compressor are shown in Table II.

In the tests we used a $180^{\circ}$ phase flip which was provided by means of a controllable p-i-n diode. The diode provided $180^{\circ}$ phase switching in approximately $5 \mathrm{~ns}$. Note that this time is comparable with round-trip time (13 ns). Nevertheless, a power gain of $G=3-4.5$ was reached with good efficiency. Typical oscillograms of the incident and compressed pulses are shown in Fig. 16. A maximum power gain of $G=4.5$ and efficiency $>60 \%$ were reached at $30 \mathrm{GHz}$ [see Fig. 16(a)] in accordance with calculation prediction. The flattop was only several nanoseconds wide because of relatively slow phase switching and too big transition time of converters in comparison with the round-trip delay time. At a lower frequency $f=$ $29.6 \mathrm{GHz}$ [see Fig. 16(b)], the pulse shape is more similar to an ideal "rectangular" form because phase switching was 2 times faster. However, $29.6 \mathrm{GHz}$ is far from optimal frequency for all converters. That is why high-power gain could not be reached in this case.

\section{CONCLUSIONS}

The proposed pulse compressor is based on a matched cavity operating with a superposition of the three lowest axis-symmetrical modes. Unlike the usual SLED-II compressor, two channels are replaced by a single channel, and need for a 3-dB coupler is avoided. Since all modes in the compressor are so-called "breakdown proof," an extremely high-power level could be achieved with this circuit.

The low-power tests that were carried out show operation of the compressor circuit with good agreement between the measured and calculated parameters.

\section{ACKNOWLEDGMENTS}

This work was supported in part by the U.S. Department of Energy, Office of High Energy Physics. Appreciation is 
expressed to the referee who brought Ref. [4] to the author's attention, and who pointed out the need to provide compensation in cavity length changes due to thermal fluctuations, as discussed in Sec. II.

[1] P. B. Wilson, SLAC-PUB-7449, 1997.

[2] P. B. Wilson, Z. D. Farkas, and R. D. Ruth, in Proceedings of the Linear Accelerator Conference, Albuquerque, 1990.

[3] S. G. Tantawi et al., Phys. Rev. ST Accel. Beams 8, 042002 (2005).

[4] S. Yu. Kazakov, Three-mode SLED-II, KEK-SLAC ISG9 Meeting, KEK, 2002, http://lcdev.kek.jp/ISG/ ISG9.Kazakov2.pdf.

[5] S. V. Kuzikov et al., in Proceedings of 6th International Workshop on Strong Microwaves in Plasmas, Nizhny Novgorod, 2005, edited by A. G. Litvak (IAP RAS, Nizhny Novgorod, 2006), Vol. 1, p. 330.
[6] S. V. Kuzikov, M.E. Plotkin, A. A. Vikharev, and J.L. Hirshfield, in Proceedings of EPAC, Edinburgh, 2006, p. 1411.

[7] M. I. Rabinovich and D.I. Trubetskov, Oscillators and Waves In Linear and Nonlinear Systems, Mathematics and its Applications (Springer, New York, 1989), Vol. 50.

[8] O. A. Nezhevenko et al., in Proceedings of EPAC, Vienna, 2000, p. 2087.

[9] I. Syratchev, G. Denisov, Vl. Kocharovsky, S. Kuzikov, and A. Stepanov, CLIC-Note-645, 2005.

[10] S. V. Kuzikov et al., in Proceedings of 7th Workshop on High Energy Density and High Power RF, Kalamata, 2005, edited by D. K. Abe and G. Nusinovich (American Institute of Physics, New York, 2006), Vol. 807, p. 463.

[11] V. P. Yakovlev, O. A. Nezhevenko, and J. L. Hirshfield, in 11th Advanced Accelerator Concepts Workshop, Stony Brook, 2004, edited by V. Yakimenko, AIP Conf. Proc. No. 737 (AIP, Melville, New York, 2004), p. 643. 